\title{
Disruption of cell wall fatty acid biosynthesis in Mycobacterium tuberculosis using a graph theoretic approach
}

\author{
Veeky Baths ${ }^{1 *}$, Utpal Roy ${ }^{1}$ and Tarkeshwar Singh ${ }^{2}$
}

\author{
* Correspondence: \\ veeky_baths@yahoo.co.in \\ 'Department of Biological Sciences, \\ Birla Institute of Technology \& \\ Science (BITS) Pilani K K BIRLA Goa \\ Campus, Goa 403 726, India \\ Full list of author information is \\ available at the end of the article
}

\begin{abstract}
Fatty acid biosynthesis of Mycobacterium tuberculosis was analyzed using graph theory and influential (impacting) proteins were identified. The graphs (digraphs) representing this biological network provide information concerning the connectivity of each protein or metabolite in a given pathway, providing an insight into the importance of various components in the pathway, and this can be quantitatively analyzed. Using a graph theoretic algorithm, the most influential set of proteins (sets of $\{1,2,3\}$, etc.), which when eliminated could cause a significant impact on the biosynthetic pathway, were identified. This set of proteins could serve as drug targets. In the present study, the metabolic network of Mycobacterium tuberculosis was constructed and the fatty acid biosynthesis pathway was analyzed for potential drug targeting. The metabolic network was constructed using the KEGG LIGAND database and subjected to graph theoretical analysis. The nearness index of a protein was used to determine the influence of the said protein on other components in the network, allowing the proteins in a pathway to be ordered according to their nearness indices. A method for identifying the most strategic nodes to target for disrupting the metabolic networks is proposed, aiding the development of new drugs to combat this deadly disease.
\end{abstract}

\section{Background}

The complete genome sequence of the best-characterized strain of Mycobacterium tuberculosis, H37Rv, has been determined and analyzed, improving understanding of the biology of this slow-growing pathogen and aiding the development of new prophylactic and therapeutic interventions [1]. The genome information concerning the H37Rv strain was used in this study.

Graph representation of the entire metabolism of the bacterium demonstrates the various clusters of proteins and their connectivity [2]. Furthermore, analyzing a wellconnected cluster of proteins linked to several pathways enables the specific pathway concerned with mycolic acid synthesis to be targeted [3]. The bacterium possesses a thick layer of lipid on the outer surface that protects it from noxious chemicals and the host's immune system [3]; these lipids are also present in the CorynebacteriumMycobacterium-Nocardia group. They give rise to important characteristics including resistance to chemical injury and dehydration, low permeability to antibiotics, virulence, acid-fast staining and the ability to persist within a host. Mycolic acids are the

(c) 2011 Baths et al; licensee BioMed Central Ltd. This is an Open Access article distributed under the terms of the Creative Commons Attribution License (http://creativecommons.org/licenses/by/2.0), which permits unrestricted use, distribution, and reproduction in any medium, provided the original work is properly cited. 
major constituents of this protective layer [4] and they play important roles as structural components of the cell wall and envelope [5]. In particular, the cyclopropane rings of mycolic acids in $M$. tuberculosis contribute to the structural integrity of the cell wall complex and protect the bacillus from oxidative stress (hydrogen peroxide) [3]. The lack of drug compliance, the appearance of multi-drug-resistant strains and the AIDS epidemic are factors that have led to a resurgence of tuberculosis infection. Drug resistance follows inadequate compliance, and AIDS patients with a weakened immune system are very susceptible to $M$. tuberculosis and it is a common cause of death [3].

Various graph theory approaches have been used to analyze metabolism in bacteria. In the present study, construction of a metabolome-based reaction network of Mycobacterium tuberculosis was attempted using the KEGG LIGAND database, and graph spectral analysis of the network was carried out to identify hubs and the sub-clustering of reactions. Another approach used for drug targeting was the identification of the 'load points' and 'choke points' in metabolic networks (graphs representing metabolism). In order to identify potential drug targets (based on the biochemical lethality of metabolic networks), the concept of choke points and load points was used to identify enzymes (edges) that uniquely consume or produce a particular metabolite (node) [6]. Complete genome sequences describe the range of metabolic reactions possible for an organism, but they cannot quantitatively describe the behavior of these reactions. In this study, a novel method for modeling metabolic states using whole cell measurements of gene expression is presented. The method, called E-Flux (a combination of Flux and Expression), extends the technique of Flux Balance Analysis by modeling maximum flux constraints as a function of measured gene expression [7].

\section{Methods}

\section{A Graph Theoretic Approach}

An ordered pair $G=(V, E)$, where $V$ is a non-empty set whose elements are called vertices (nodes or points), and $\mathrm{E}$ is a set of two distinct elements that are a subset of $\mathrm{V}$, whose elements are called edges (links or lines) [8]. Furthermore, a graph $\mathrm{G}$ is said to be finite if $\mathrm{V}$ is finite, otherwise it is termed infinite. Two vertices, $\mathrm{u}$ and $\mathrm{v}$, are said to be adjacent in $\mathrm{G}$ if $\mathrm{u}$ and $\mathrm{v}$ are joined by an edge, otherwise they are non-adjacent. An edge e and a vertex $u$ are said to be incident if $u$ is one of the end vertices of the edge e. Two edges, $e_{1}$ and $e_{2}$, are said to be adjacent if both have the same end vertices. The cardinality of $\mathrm{V}$ is denoted by $|\mathrm{V}|=\mathrm{p}$ : = number of vertices in $\mathrm{V}$, is called the order of the graph G. Similarly, the cardinality of $E$ i.e. $|E|=q$ is called the size of G. The graph can be represented using diagrams and matrices. On the basis of adjacency and incidence relationships among edges and vertices, adjacency and incidence matrices can be determined [8].

The adjacency matrix of a graph $G$, with vertex set $V=\left\{v_{1}, v_{2}, \ldots, v_{p}\right\}$, is denoted $A(G)$ $=\left[a_{i, j}\right]_{p} \times p$, where $a_{i, j}=1$ if there is an edge between $v_{i}$ to $v_{j}$ and 0 otherwise. It is a binary $(0,1)$ - square symmetric matrix. The incidence matrix of a graph $G$ is denoted $I(G)=\left[b_{i, j}\right]_{p \times q}$, where $b_{i, j}=1$ if $e_{i}$ is incident to $v_{i}$, and 0 otherwise. A graph $\mathrm{H}=$ $\left(V_{1}, E_{1}\right)$ is said to be a sub-graph of the graph $G=(V, E)$ if $V_{1}$ is a subset of $V$ and $E_{1}$ is a subset of $E$. When $V_{1}=V$ then the sub-graph $H$ is termed a spanning sub-graph of $\mathrm{G}$. A directed graph (digraph in short) $\mathrm{D}$ is an ordered pair $\mathrm{D}=(\mathrm{V}, \mathrm{A})$, where $\mathrm{V}$ is 
known as a vertex set of $\mathrm{D}$ and $\mathrm{A}$ is an ordered pair of two distinct elements of $\mathrm{V}$, known as an arc set of $\mathrm{D}$. The order and size of a digraph $\mathrm{D}$ is the number of vertices and $\operatorname{arcs}$ in $D$, respectively. In a digraph, if there is an $\operatorname{arc} a=(u, v)$, then $u$ is the initial vertex and $v$ is the terminal vertex of the arc a. A graph $G$ is said to be connected if one vertex can be reached from another vertex by a path (alternating sequence of vertices and edges, i.e. $u=u_{0}, e_{0}, u_{1}, e_{1}, u_{2}, \ldots, u_{n-1}, e_{n-1}, u_{n}=v$ ), otherwise it is considered to be disconnected.

A digraph is said to be strongly connected if a vertex of $\mathrm{D}$ can be reached from other vertices of $\mathrm{D}$ by a directed path. A digraph $\mathrm{D}$ is said to be weakly connected if its underlying graph (undirected graph) is connected. Deletion of a vertex $v$ from the digraph $D$ (graph G) refers to the removal of a vertex $\mathrm{v}$ from $\mathrm{V}$ and all arcs (edges) for which $\mathrm{v}$ is either the initial vertex or the terminal vertex of an arc in D. Deletion of an arc (edge) from a digraph (graph) refers to the removal of the arc (edge) from the digraph (graph). If $\mathrm{S}$ is a subset of the vertex set $\mathrm{V}$ of the digraph (graph), then $\mathrm{D}-\mathrm{S}$ is the digraph (graph) obtained by removing vertices of $\mathrm{S}$ and arcs (edges) for which one of the end vertices are in $S$ from the $D$. If there are more than two connected components, then $S$ is referred to as the separating set of D. Let $[S, V-S]=\{(\mathrm{u}, \mathrm{v}): \mathrm{u} \varepsilon \mathrm{S}$ and $\mathrm{v} \varepsilon \mathrm{V}-\mathrm{S}\}$; if all arcs (edges) of [S, V-S] are removed from the digraph (graph) and the resultant digraph (graph) is not connected, then [S, V-S] is termed an edge-cut of D.

The mycolic acid network [9] was modeled using a digraph (Figure 1) in which vertices represent the metabolites and reactions/interactions between any two metabolites are represented by arcs (Figure 2). Let $\mathrm{D}=(\mathrm{V}, \mathrm{A})$ be the digraph with vertex set $\mathrm{V}=$ $\left\{\mathrm{v}_{1}, \mathrm{v}_{2}, \mathrm{v}_{3}, \ldots, \mathrm{v}_{49}\right\}$ and there are four other metabolic cycles connected to the vertices $\mathrm{v}_{1}, \mathrm{v}_{10}$ and $\mathrm{v}_{40}$ of the digraph $\mathrm{D}$ and $\operatorname{arc}$ set $\mathrm{A}=\left\{\mathrm{e}_{1}, \mathrm{e}_{2}, \mathrm{e}_{3}, \ldots, \mathrm{e}_{80}\right\}$. By selecting the vertices with maximum out-degree (i.e. number of arcs radiating from the vertex) first, a set $S=\left\{v_{11}, v_{10}, v_{39}, v_{15}, v_{19}, v_{24}, v_{29}, v_{34}, v_{46}\right\}$ can be generated in D. Deleting this set

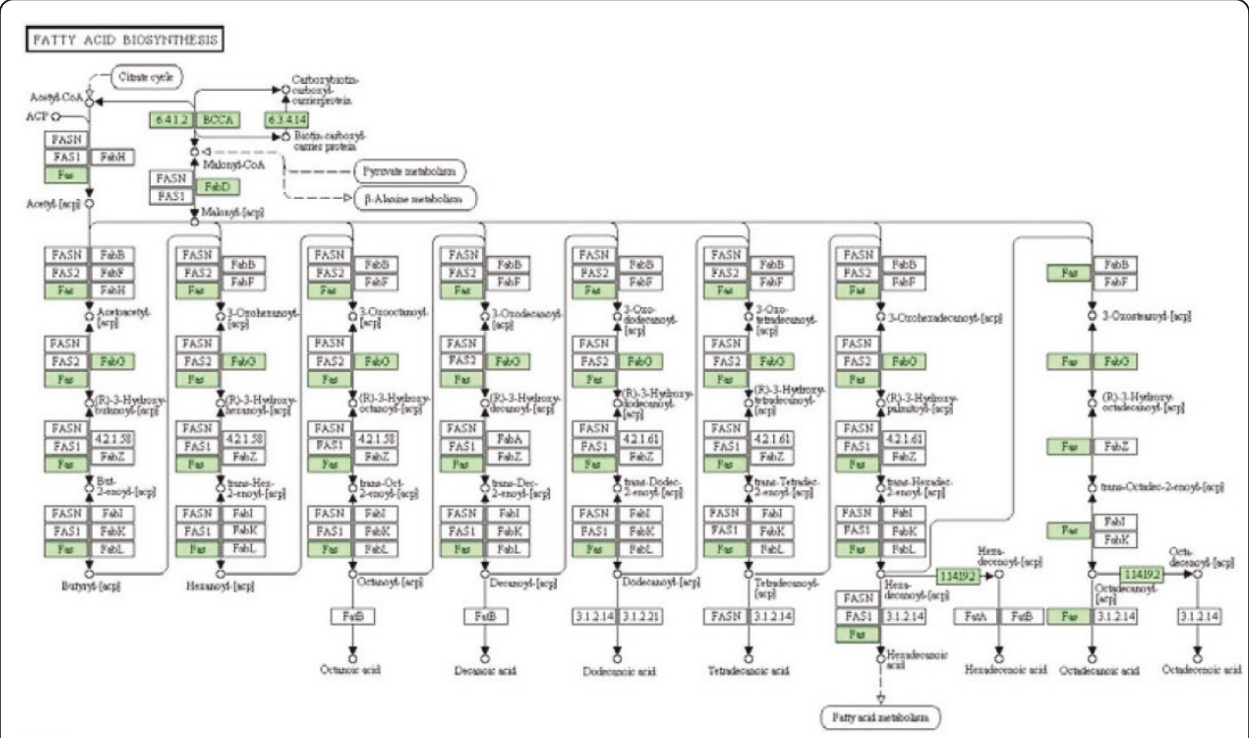

Figure 1 Fatty acid Biosynthesis (Source KEGG pathway database). Fatty Acid Synthesis Pathway in Mycobacterium tuberculosis H37rv. The pathway was downloaded from the KEGG Database. Starting from Acetyl-CoA at the bottom left of the figure, the metabolites are numbered left to right and then bottom to top. 


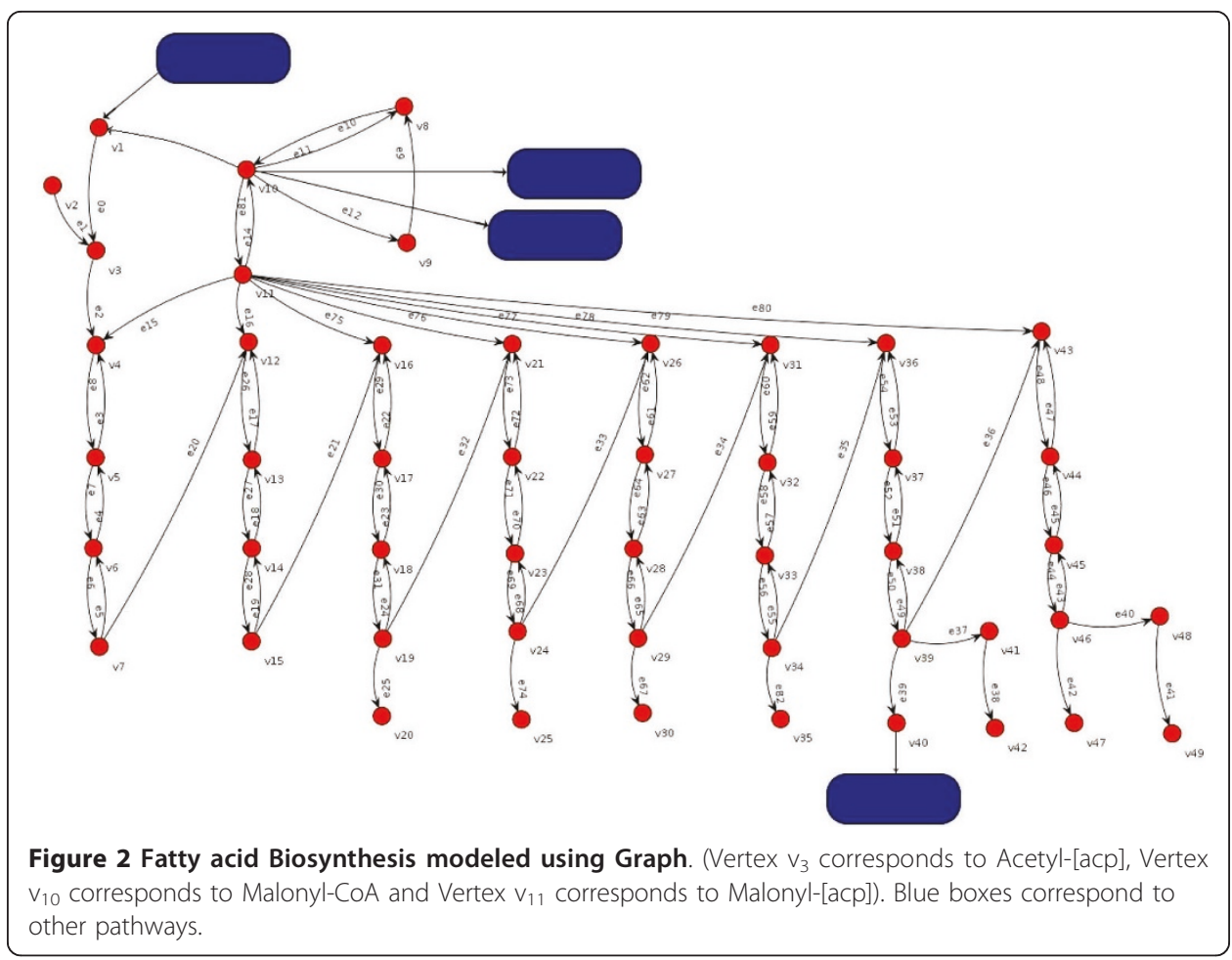

$\mathrm{S}$ from the digraph $\mathrm{D}$ refers to deleting all the vertices of $\mathrm{S}$ from $\mathrm{D}$ and $\operatorname{arcs}$ that have one end vertex in S. Let $\mathrm{D}^{*}=\left(\mathrm{V}^{*}, \mathrm{~A}^{*}\right)$ be the resultant digraph obtained, where $\mathrm{V}^{*}=$ $\mathrm{V}-\mathrm{S}$ and $\mathrm{A}^{*}$ is the set of all arcs remaining in $\mathrm{A}$, which has more than four components in $\mathrm{D}^{*}$, a disconnected digraph.

\section{Determination of nearness index}

The nearness index for a vertex (protein) is the sum of all the inverses of the minimum path lengths to every other vertex in the graph [2]. For a particular vertex $\mathbf{v}_{\mathbf{i}}$, the eccentricity of $v_{i}$ is the length of the path from the farthest vertex in the graph and would contribute least to the nearness index of $\mathbf{v}_{\mathbf{i}}$ because the inverse of the path length is added equivalently; vertices with more eccentricity have a lower nearness index. If the degree of vertex $\mathbf{v}_{\mathbf{i}}\left(\mathbf{i}^{\text {th }}\right.$ vertex of given graph $\left.\mathbf{G}\right)$ is denoted by $\mathbf{d}_{\mathbf{i}}$, then the nearness index of $\mathbf{v}_{\mathbf{i}}$ is given by:

$$
\mathrm{N}_{\mathrm{i}}=\Sigma\left(1 / \mathrm{d}_{\mathrm{i}}\right)
$$

The data were parsed using a java program that uses the list of minimum path lengths (obtained from visANT) as the input.

\section{Results}

Data for assessing the pathway are available in the KEGG (Kyoto Encyclopedia of Genes and Genomes) pathway database and were used to obtain a flowchart of the fatty acid biosynthesis pathway of Mycobacterium tuberculosis H37Rv. The green squares in the flowchart (Figure 1) represent proteins identified from the genome sequence of the bacterium. The genome sequence for this bacterium is complete. Therefore, the information concerning the participating proteins is also complete [10]. 
The proteins involved in the mycolic acid biosynthesis pathway [9] were inputted into visANT software [11]. This free software can be used to identify interactions between proteins and the result is displayed as a graph. visANT can be downloaded from http://visant.bu.edu/. Programming for assessing the results was carried out using java (version 1.6.0_01). NetBeans IDE 6.9.1 was the IDE (Integrated Development Environment) used [12]. The name of the organism was selected and the list of proteins entered in the input area provided. Clicking the search button provides the interactions among the proteins given as the input. A graph is obtained and when the layout "spring embedded" is selected, the following is produced: Figure 3.

visANT represents proteins as vertices and the interactions between them as edges. Therefore, an undirected graph of the interactions of proteins involved in the fatty acid biosynthesis pathway is obtained. The graph is not directed as demonstrated in Figure 4.

This result as shown in Figure 5. can be stored in a text file and parsed to obtain the path distance (starting protein and the ending protein). For example, for the line Shortest path (2)::RV1384-RV2967C-RV0263C, the shortest path from protein RV1384 to protein RV0263C, is of length 2 . This datum can be used to obtain the degree of each vertex. Therefore, when all the degrees have been determined, the amount of influence a protein possesses, using the concept of nearness index, can be calculated.

\section{Nearness index}

The nearest vertex (with small ecentricity) would contribute most to the nearness index. Therefore, when calculating the nearness index, all the vertices of the graph were taken into account as were their differing levels of influence. The total sum represents the influence of the protein represented by $\mathbf{v}_{\mathbf{i}}$ on the complete pathway, which is

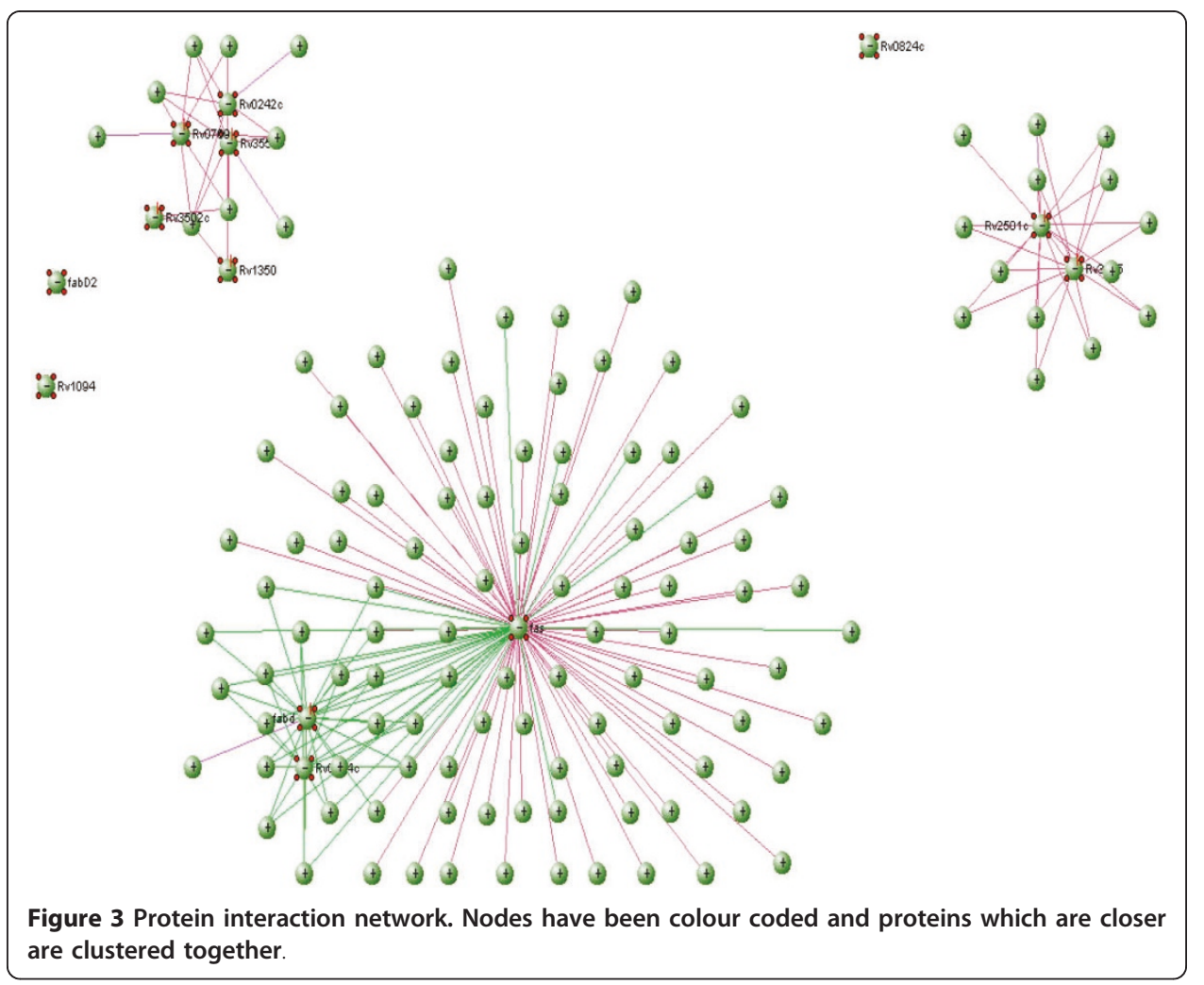




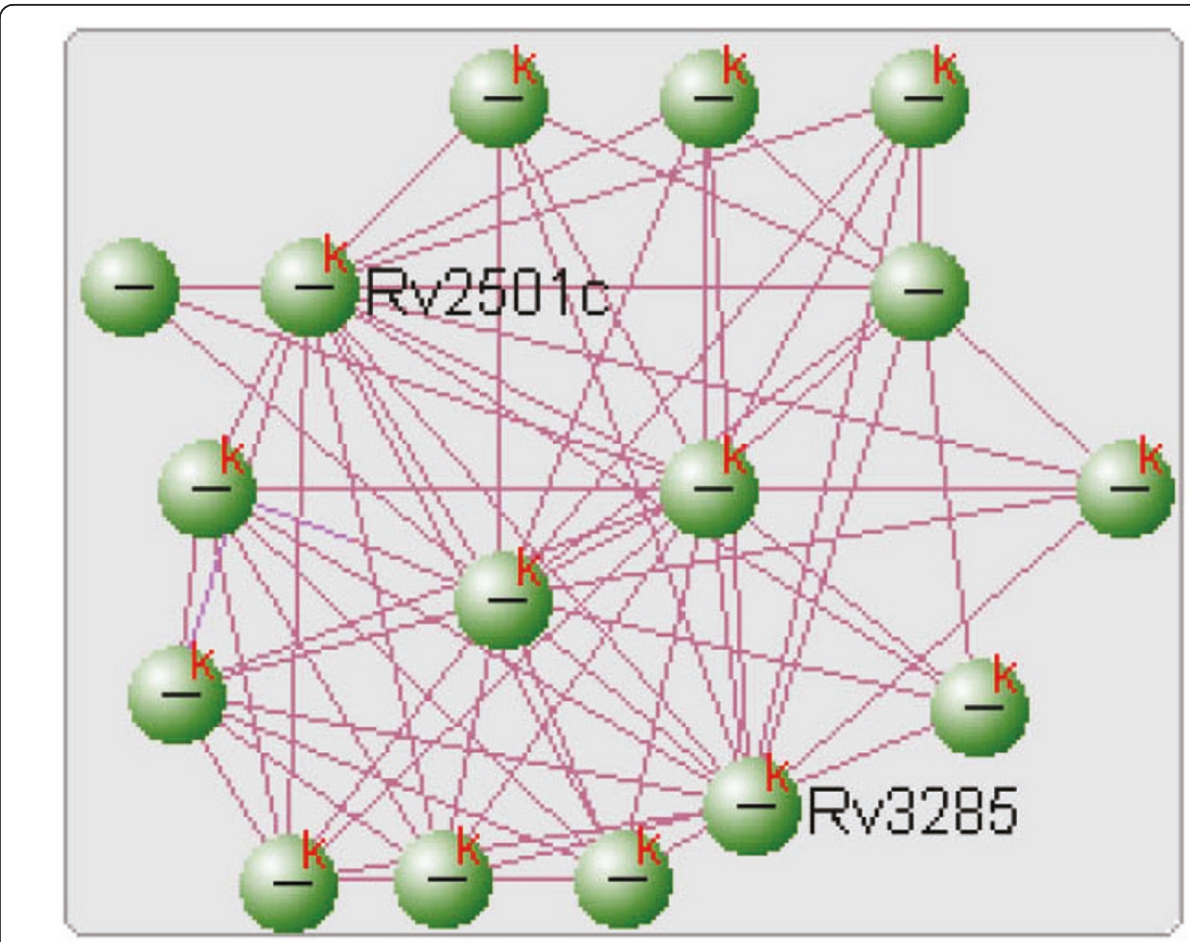

Figure 4 Protein network demonstrating dense inter-connectivity. visANT possesses a tool to identify the shortest paths between all the pairs of vertices in a graph. Selecting all the vertices of this small graph identifies the shortest paths. The shortest paths are given in the following format:

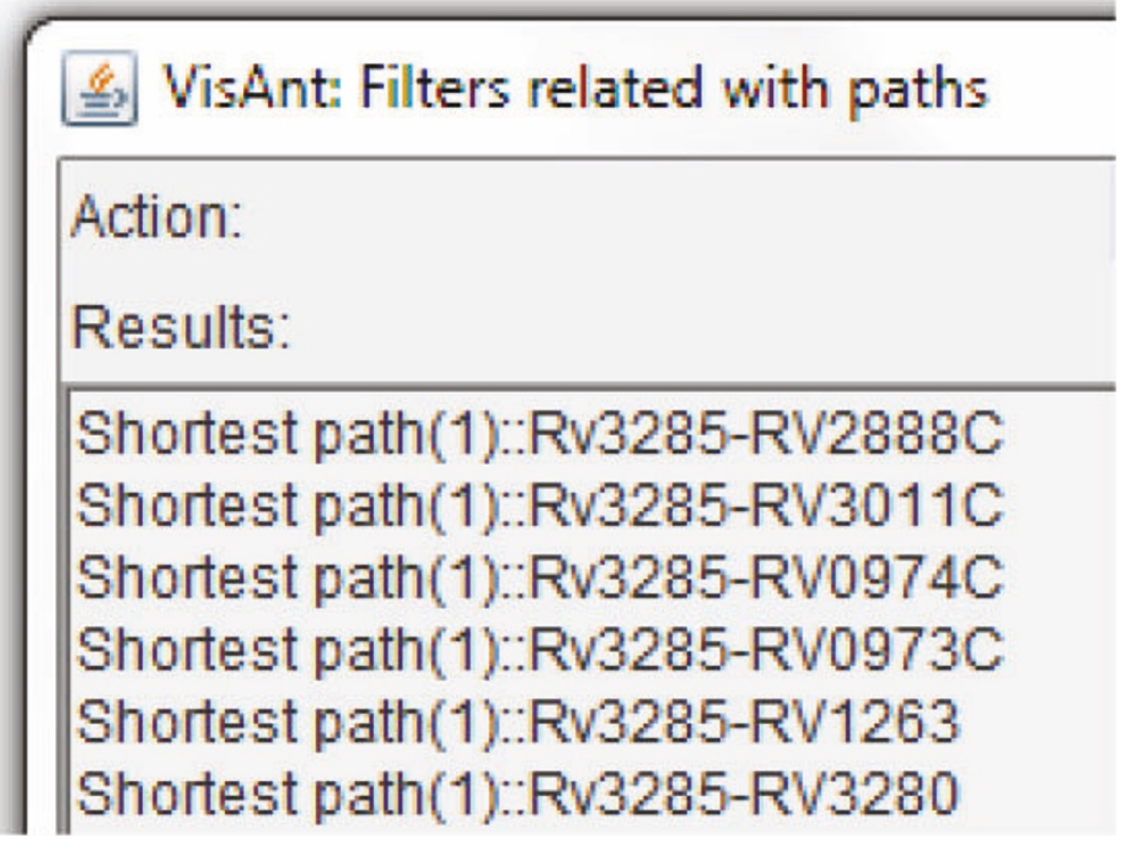

Figure 5 Shortest Pathway analysis. The shortest paths between all the pairs of vertices in a graph (Source: visANT). 
represented as a graph. Another interaction between proteins concerns one protein helping in the production of a metabolite, which is converted to another metabolite by a second protein (2). In this case, the first protein influences the second protein. The second protein relies on the first protein functioning so that it obtains the metabolites. The graph obtained from protein-protein influences of this kind will be a directed graph. Reversible reactions are represented as edges to and from the two proteins. From this, the influence of each protein can be estimated by calculating its nearness index (Table 1). The result of shortest paths obtained from visANT can be copied and stored in a file called shortpath, which is given as an argument for running the java program. A java code like that shown below was written to calculate the nearness index with the file shortpath as the input.

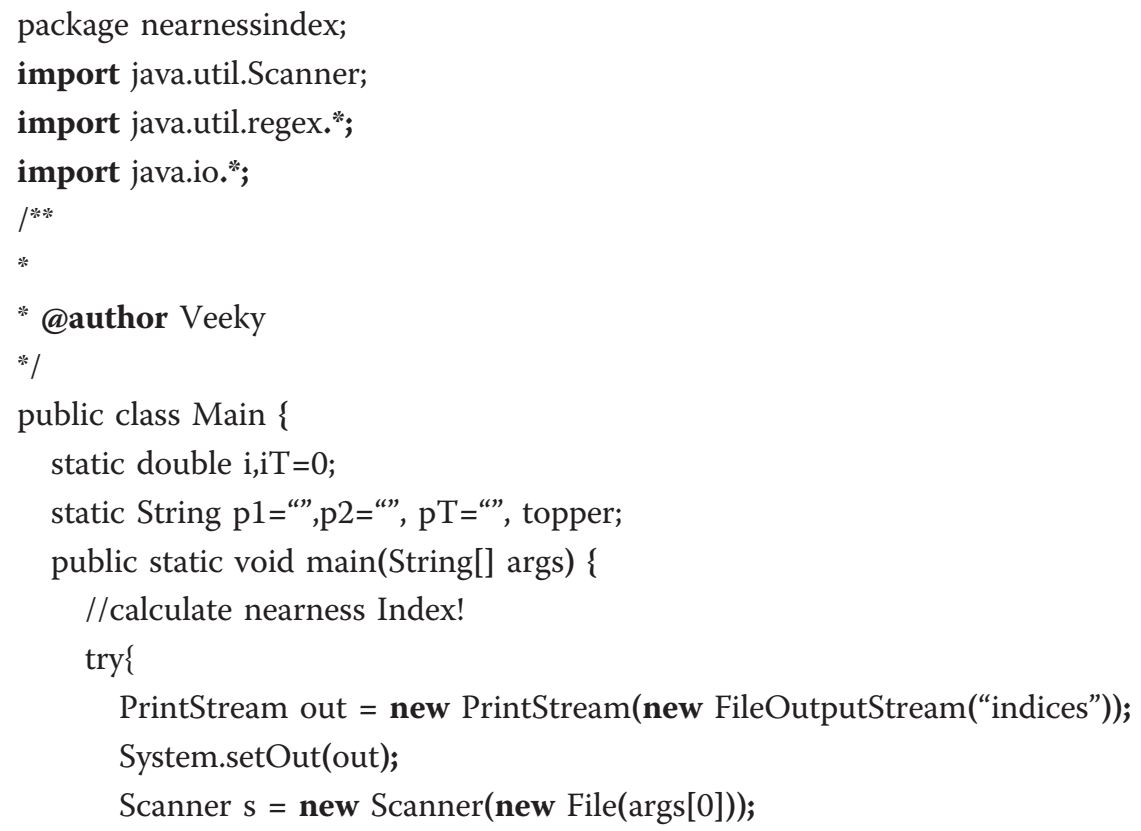

Table 1 Protein nearness index

\begin{tabular}{ll}
\hline Protein & $N_{i}$ (Nearness Index) \\
\hline RV2501C & 15 \\
\hline RV2967C & 15 \\
\hline RV0973C & 15 \\
\hline Rv3285 & 14.5 \\
\hline RV0263C & 12 \\
\hline RV3280 & 11.5 \\
\hline RV2502C & 11.5 \\
\hline RV3799C & 11.5 \\
\hline RV0974C & 11.5 \\
\hline RV2247 & 11.5 \\
\hline RV2888C & 10 \\
\hline RV3375 & 10 \\
\hline RV3011C & 10 \\
\hline RV1263 & 10 \\
\hline RV2363 & 10 \\
\hline RV1384 & 9 \\
\hline
\end{tabular}


while (s.hasNextLine()) \{

s.findInLine(“Shortest path $\backslash\left(((\backslash \mathrm{d}+) \backslash \backslash)::(\backslash \mathrm{w}+) .{ }^{*}-(\backslash \backslash \mathrm{w}+)\right.$ ));//at each line, look for this pattern

MatchResult result $=\mathrm{s} \cdot \mathrm{match}() ; / /$ results from

if ((p1.equals(result.group(2)))\&\&(!p2.equals(result.group(3)))) \{

$\mathrm{i}=\mathrm{i}+(1 /$ Double.parseDouble(result.group(1)));

p2 = result.group $(3)$;

\}

else if (!p1.equals(result.group(2))) \{

if (!p1.equals("')) \{

out.println(p1+ ": " + i);

if $(\mathrm{iT}<\mathrm{i})\{$

topper $=\mathrm{p} 1 ; / /$ topper is the string that would hold the proteins with

highest nearness index

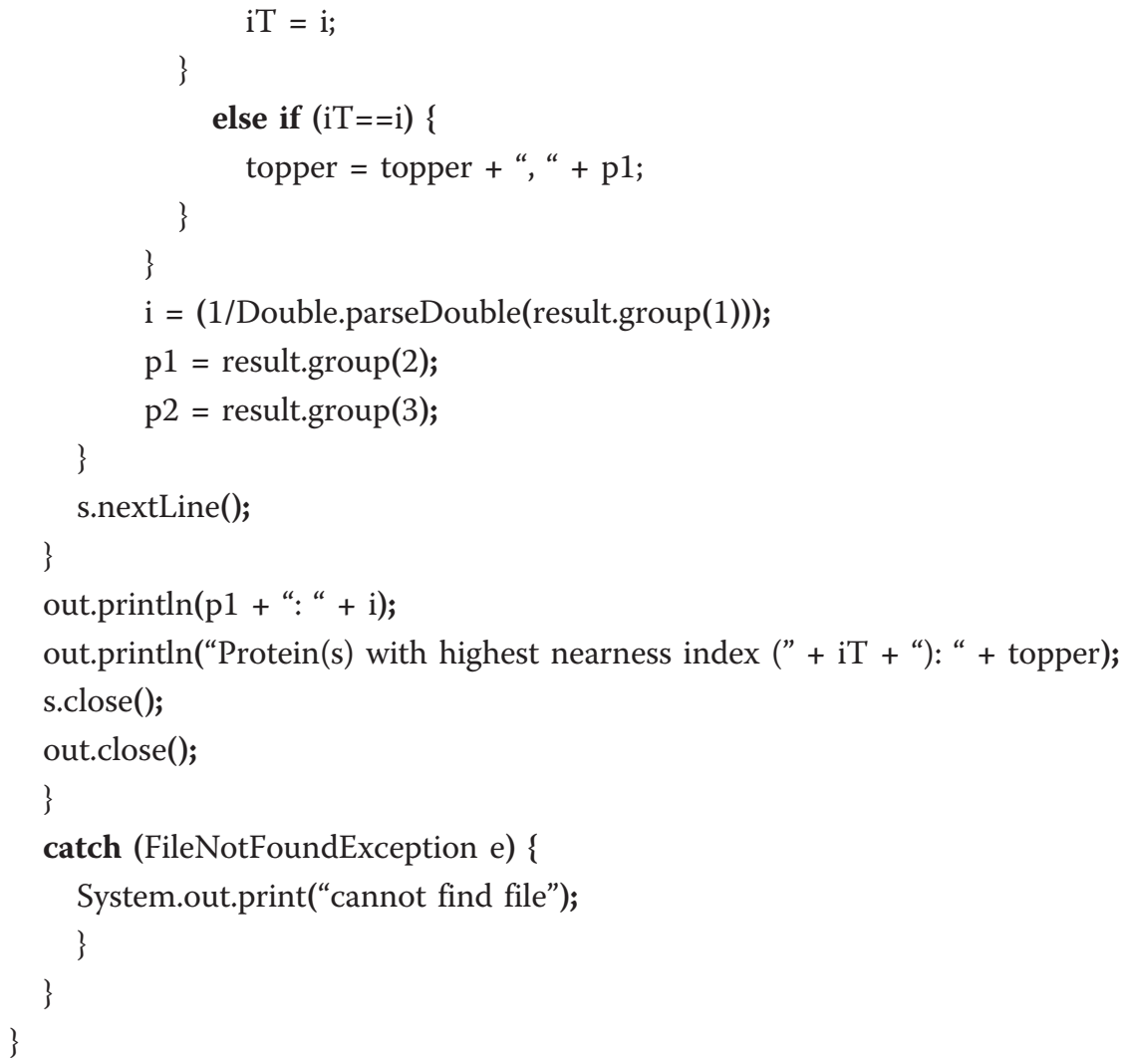

\section{Computation of nearness index with Java code}

In the main method of the program, a new PrintStream object was used to print to an output file called indices. A scanner object was used to read from the input file, shortpath, line by line. A while loop was used to check if shortpath was read up to the last line.

Inside the while loop, for each line a regex function (Shortest path $\backslash((\backslash \backslash d+) \backslash \backslash)::(\backslash \mid w+)$. *-( $(\backslash w+))$ was used to identify the starting protein of the path, the ending protein and the path length. For instance, for the line Shortest path(2)::RV1384-RV2967CRV0263C, we would obtain the result group(1) as 2, result group(2) as RV1384 and 
result group(3) as RV0263C. The shortest paths from each protein were calculated and according to the formula for the nearness index, the inverses of the shortest path length could be added. Redundant data concerning the various paths from one protein to another were discarded:

Shortest path(2)::RV2380C-RV2246-RV0099

Shortest path(2)::RV2380C-RV2245-RV0099

Shortest path(2)::RV2380C-RV1454C-RV0099

Shortest path(2)::RV2380C-RV0149-RV0099

Shortest path(2)::RV2380C-RV2381C-RV0099

Therefore, only one of these paths was considered as they were all considered to be the shortest.

Using visANT, the shortest path from each protein in to all the other proteins (of the form Shortest path(2)::RV1384-RV2967C-RV0263C) were obtained. This shortest path was used as an input for the java program to calculate the nearness index presented in Table 1.

The proteins in Figure 6 with the highest nearness index of 15 were RV2501C, RV2967C and RV0973C. According to computational factors (namely domain fusion, gene neighborhood and phylogenetic profiling), these were the most influential proteins in this particular sub-graph. Among these proteins, RV2501C is directly involved in the fatty acid biosynthesis.

For the largest sub-graph (Figure 7), the proteins with the largest nearness index of 104.4167 were RV2524C (FAS), RV2245 and RV2246. Of these proteins, FAS is directly involved in the pathway as shown in Table 2 .

\section{Discussion}

One of the pathways of Mycobacterium tuberculosis concerns fatty acid biosynthesis, and contributes to the synthesis of mycolic acid. The outer lipid layer (cell wall) of the bacterium makes it difficult for broad spectrum antibiotics to have any effect [4], and a major component of the cell wall is mycolic acid. Therefore, when synthesis of mycolic acid is reduced, broad spectrum antibiotics would be more effective owing to cell wall
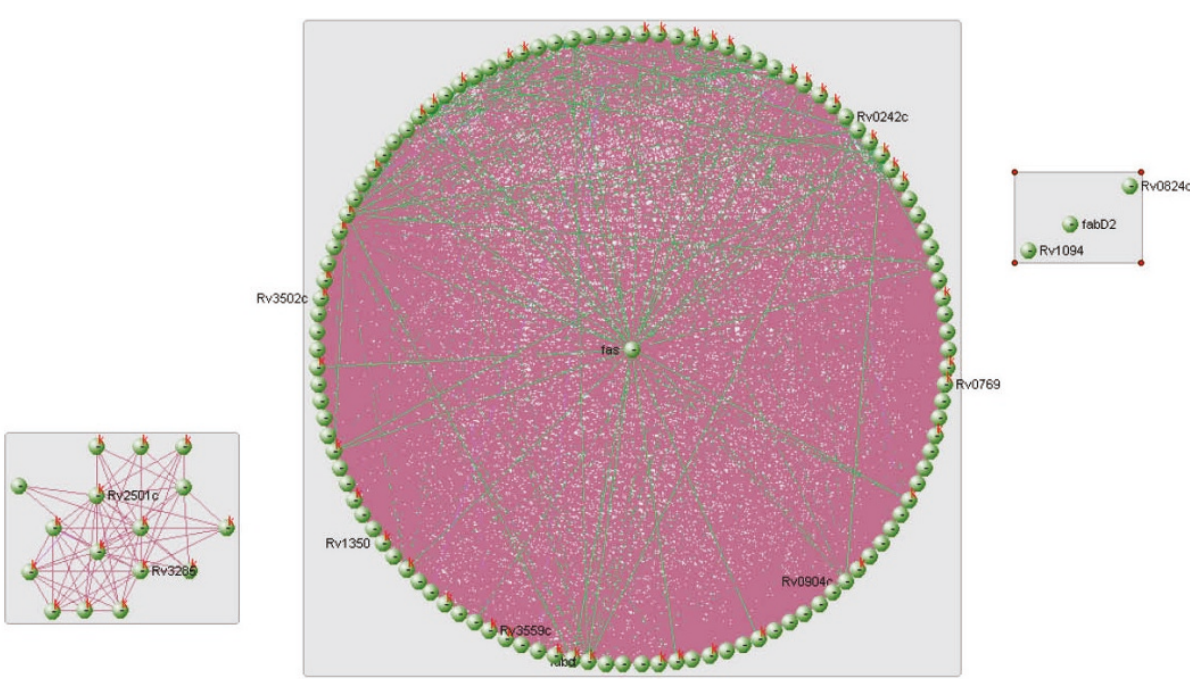

Figure 6 Protein networks and their interactions (Source: visANT). 


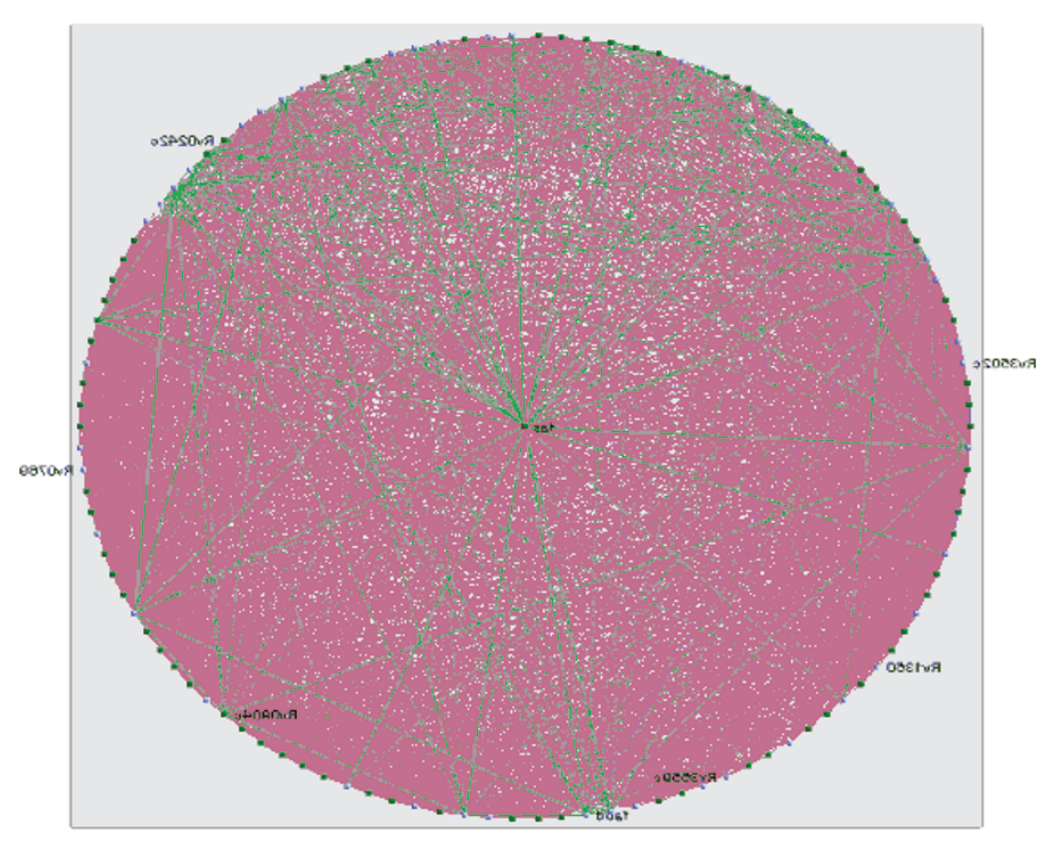

Figure 7 The largest sub-graph. This graph was used to calculate the nearness index of proteins.

damage. Mycolic acid synthesis is the target of well-known anti-tuberculosis drugs including isoniazid, ethionamide and thiocarlide [9]. This suggests that any reactions that contribute to synthesis and processing of mycolic acids are viable targets for new drug discovery. visANT represents proteins as vertices and the interactions between them as edges, and there are various interactions between proteins involved in the fatty acid biosynthesis pathway.

Ranking proteins by the topological properties of the human protein-protein interaction net-work is one strategy for drug-target identification [13]. Another approach characterizes the interaction properties in protein-protein complexes; for example, identifying the domains involved in binding or analyzing the 3D structure. Comparison of domain-domain interactions and interfaces across an interactome can identify selective drug targets or drugs targeting multiple proteins (to block parallel pathways in a network) [14]. Structural analysis can be carried out to identify pockets where drugs could bind and to compare their properties with binding pockets on other proteins in the network [15].

The shortest and alternate paths in the reaction networks were examined. In an earlier study, sub-cluster profiling demonstrated that reactions in the mycolic acid pathway of mycobacteria form a tightly connected sub-cluster. Identification of hubs revealed that reactions involving glutamate were central to mycobacterial metabolism, and those involving pyruvate were at the centre of the E. coli metabolome. The analysis of shortest paths between reactions has revealed several paths that are shorter than well-established pathways. Using a directed graph to represent pathways would enable researchers to determine the importance of various proteins in a pathway and how their removal would affect that pathway [11].

The graph nodes represent metabolites and the edges represent enzymes. Based on an extended form of the graph theory model of metabolic networks, metabolite 
Table 2 Nearness Index For the largest sub-graph

\begin{tabular}{|c|c|c|c|c|c|}
\hline Protein & $\begin{array}{l}N_{i} \text { (Nearness } \\
\text { Index) }\end{array}$ & Protein & $\begin{array}{l}N_{i} \text { (Nearness } \\
\text { Index) }\end{array}$ & Protein & $\begin{array}{l}N_{i} \text { (Nearness } \\
\text { Index) }\end{array}$ \\
\hline RV2524C & 104.4167 & RV3826 & 75.25 & RV0533C & 60.85 \\
\hline RV2245 & 101.4167 & RV1925 & 74.75 & RV1108C & 60.35 \\
\hline RV2246 & 100.9167 & RV2941 & 74.75 & RV3246C & 59.35 \\
\hline RV2048C & 91.25 & RV0166 & 74.75 & RV2884 & 59.35 \\
\hline RV2935 & 91.25 & RV3801C & 74.75 & RV3765C & 59.35 \\
\hline RV2934 & 91.25 & RV1427C & 74.75 & RV0903C & 59.35 \\
\hline RV2933 & 91.25 & RV1058 & 74.75 & RV0491 & 59.35 \\
\hline RV2932 & 91.25 & RV3515C & 74.75 & RV1027C & 59.35 \\
\hline RV2931 & 91.25 & RV1206 & 74.75 & RV0981 & 59.35 \\
\hline RV3825C & 91.25 & RV2383C & 74.68333 & RV0757 & 59.35 \\
\hline RV2940C & 91.25 & RV3561 & 72.25 & RV0602C & 59.35 \\
\hline RV3800C & 91.25 & RV1185C & 71.75 & RV1033C & 59.35 \\
\hline RV1181 & 91.25 & RV2948C & 71.75 & RV0818 & 59.35 \\
\hline RV0405 & 91.25 & RV2930 & 71.75 & RV2783C & 58.68333 \\
\hline RV1527C & 91.25 & RV0035 & 71.75 & RV2727C & 58.68333 \\
\hline RV1664 & 91.25 & RV2950C & 71.75 & RV3907C & 58.18333 \\
\hline RV1662 & 91.25 & RV1193 & 71.75 & RV3105C & 58.18333 \\
\hline RV1661 & 91.25 & RV0404 & 71.75 & RV1630 & 58.18333 \\
\hline RV2381C & 90.75 & RV1013 & 71.75 & RV2539C & 56.85 \\
\hline RV2048C_12 & 90.75 & RV3667 & 71.68333 & RV3607C & 56.35 \\
\hline RV2946C & 90.75 & RV2590 & 71.68333 & RV1409 & 56.35 \\
\hline RV2382C & 89.08333 & RV2505C & 71.25 & RV2244 & 54.18333 \\
\hline RV2947C & 89.08333 & RV3506 & 71.25 & RV2214C & 54.08333 \\
\hline RV1180 & 89.08333 & RV1750C & 71.25 & RV3389C & 53.08333 \\
\hline RV1483 & 87.33333 & RV0542C & 71.25 & RV3538 & 53.08333 \\
\hline RV1663 & 86.18333 & RV0551C & 71.25 & RV1938 & 51.75 \\
\hline RV3141 & 84.68333 & RV1345 & 71.25 & RV3617 & 51.75 \\
\hline RV3777 & 84.68333 & RV2384 & 70.18333 & RV0134 & 51.75 \\
\hline RV0149 & 84.68333 & RV2380C & 69.68333 & ACCD3 & 47.33333 \\
\hline RV1454C & 84.68333 & RV0101 & 69.68333 & RV0769 & 39.78333 \\
\hline RV1912C & 84.68333 & RV3513C & 68.75 & RV3559C & 39.78333 \\
\hline RV2048C_11 & 80.18333 & RV2187 & 68.68333 & RV0242C & 39.78333 \\
\hline RV0099 & 77.25 & RV1550 & 68.68333 & RV3502C & 36.61667 \\
\hline RV0214 & 77.25 & RV2379C & 66.68333 & RV1350 & 36.61667 \\
\hline RV1529 & 76.75 & fabd & 64.85 & RV0768 & 28.93333 \\
\hline RV3089 & 76.25 & RV2457C & 63.85 & RV0241C & 28.93333 \\
\hline RV0270 & 76.25 & RV3606C & 63.35 & RV3560C & 28.93333 \\
\hline RV0119 & 76.25 & RV1629 & 63.35 & & \\
\hline RV1521 & 76.25 & RV1018C & 60.85 & & \\
\hline
\end{tabular}

structural information was used to calculate the k-shortest paths between metabolites (the presence of more than one competing path between substrate and product). On the basis of these paths and connectivity information, load points were calculated and used empirically to rank the importance of metabolites/enzymes in the metabolic 
network. The load point analysis emphasized the role that the biochemical structure of a metabolite, rather than its connectivity (hubs), plays in the conversion pathway.

\section{Conclusions and scope}

The graph of the fatty acid biosynthesis of Mycobacterium tuberculosis H37Rv had two major sub-graphs. The largest sub-graph had greater influence and its proteins would be better drug targets than the smaller sub-graph. When analyzed, this large sub-graph demonstrated that the protein that most influences the pathway is FAS (Fatty Acid Synthase).

As demonstrated in Figure 2, the nearness indices of the proteins involved in the digraph can be determined. This process can be carried out on the directed graph obtained from protein dependences based on metabolites. For this, using visANT would require a line of input for each edge and this would be a cumbersome task. Therefore, alternative software such as Cytoscape is utilized. The KEGGraph library of $\mathrm{R}$ language provided by Bioconductor can be used to assess KEGG pathways in the form of graphs. This library takes KGML data for a given pathway and parses it to provide graphs. Using the resources available through visANT, the data of any biological pathway that is present in the KEGG database can be analyzed, and using a simple java program the most influential proteins in the pathway can be identified in silico.

\section{Acknowledgements}

We would like to thank our host organization Bits-Pilani K.K.Birla Goa Campus for providing us facilities and resources for carrying out this research.

\section{Author details}

${ }^{1}$ Department of Biological Sciences, Birla Institute of Technology \& Science (BITS) Pilani K K BIRLA Goa Campus, Goa 403 726, India. ${ }^{2}$ Department of Mathematics, Birla Institute of Technology \& Science (BITS) Pilani K K BIRLA Goa Campus, Goa 403 726, India.

\section{Authors' contributions}

VB contributed in pathway modelling, programming and applying graphs in biology. UR analysed the biological data. TK was involved in applying graph theoretic approach. All authors read and approved the final manuscript.

\section{Competing interests}

The authors declare that they have no competing interests.

Received: 6 January 2011 Accepted: 31 March 2011 Published: 31 March 2011

\section{References}

1. Cole ST, Brosch R, Parkhill J, Garnier T, Churcher C, Harris D, Gordon SV, Eiglmeier K, Gas S, Barry CE, Tekaia F, Badcock K, Basham D, Brown D, Chillingworth T, Connor R, Davies R, Devlin K, Feltwell T, Gentles S, Hamlin N, Holroyd S, Hornsby T, Jagels K, Krogh A, McLean J, Moule S, Murphy L, Oliver K, Osborne J, Quail MA, Rajandream MA, Rogers J, Rutter S, Seeger K, Skelton J, Squares R, Squares S, Sulston JE, Taylor K, Whitehead S, Barrell BG: Deciphering the biology of Micobacterium tuberculosis from the complete genome sequence. Nature 1998, 393:537-544.

2. Karthik R, Vashisht R, Nagasuma C: Strategies for efficient disruption of metabolism in Mycobaterium Tuberculosis from network analysis. Mol BioSyst 2009, 5:1740-1751.

3. Colijn C, Brandes A, Zucker J: Interpreting expression data with metabolic flux models: predicting Mycobacterium tuberculosis mycolic acid production. PLoS Comput Biol 2009, 5(8):e1000489.

4. Winder FG: Mode of action of the antimycobacterial agents and associated aspects of the molecular biology of the mycobactria. In The biology of the mycobacteria, Physiology, identification and classification. Volume 1. Academic Press, N. Y; 1982:353-438.

5. Takayama K, Wang C, Gurdyal SB: Pathway to Synthesis and Processing of Mycolic Acids in Mycobacterium tuberculosis. Clinic Microbiol Rev 2005, 18:81-101.

6. Syed AR, Schomburg D: Observing local and global properties of metabolic pathways: 'load points' and 'choke points' in the metabolic networks. Bioinformatics 2006, 22:1767-1774.

7. Yuan Y, Zhu Y, Crane D, Barry C: The effect of oxygenated mycolic acid composition on cell wall function and macrophage growth in Mycobacterium tuberculosis. Mol Microbiol 1998, 29:1449-1458.

8. West DB: Introduction to Graph Theory New York: Prentice Hall Publication; 2009.

9. Karthik R, Rajagopalan P, Nagasuma C: Hallmarks of mycolic acid biosynthesis: A comparative genomics study. Proteins 2007, 69:358-368 
10. The KEGG databases at GenomeNet. [http://www.genome.jp/kegg/].

11. Hu Z, Mellor J, Wu J, DeLisi C: VisANT: data-integrating visual framework for biological networks and modules. Nucl Acids Res 2005, 33:W352-W357.

12. Java web applications. [http://netbeans.org/].

13. Zhu M, Gao L, Li X, Liu Z, Xu C, Yan Y, Walker E, Jiang W, Su B, Chen X, Lin H: The analysis of the drug-targets based on the topological properties in the human protein-protein interaction network. J Drug Target 2009, 17:524-532.

14. Keskin O, Gursoy A, Ma B, Nussinov R: Towards drugs targeting multiple proteins in a systems biology approach Curr Top Med Chem 2007, 943-951.

15. Nobuyoshi S, Kazuyoshi I, Toshiyuki T, Shizu T, Jun O, Yoshiko I, Akiko S, Atsushi T, Hideki N, Tadayuki T, Satoru K, Yoshiyuki S, Takao I: An integrative in silico approach for discovering candidates for drug-targetable protein-protein interactions in interactome data. BMC Pharmacol 2007, 7:10.

doi:10.1186/1742-4682-8-5

Cite this article as: Baths et al:: Disruption of cell wall fatty acid biosynthesis in Mycobacterium tuberculosis using a graph theoretic approach. Theoretical Biology and Medical Modelling 2011 8:5.

Submit your next manuscript to BioMed Central and take full advantage of:

- Convenient online submission

- Thorough peer review

- No space constraints or color figure charges

- Immediate publication on acceptance

- Inclusion in PubMed, CAS, Scopus and Google Scholar

- Research which is freely available for redistribution 